\title{
Rheology effect determination of the heterogeneous rock massif on the stress-strain state of the breakage face geomechanical system
}

\author{
Vadym Fomychov ${ }^{1}$, Viktor Pochepov ${ }^{1}$, Liudmyla Fomychova ${ }^{1}$, and Viktor Lapko ${ }^{1 *}$ \\ ${ }^{1}$ National Mining University, Department of Underground Mining, 19 Yavornytskoho Ave., 49005 \\ Dnipro, Ukraine
}

\begin{abstract}
The purpose of this study is to determine the nature of influence of the rheological properties of the rocks on the results of computational experiments simulating the mine workings contour condition during the breakage face advance. To determine the deformations along the mine workings contour, the calculations have been made of the junction area between the preparatory and stope face mine workings by the finite element method for various parameters of the material models. These data provided the basis for analysis of the patterns development of the rheology modelling influence on the stress-strain state of the geomechanical system. The obtained patterns are described by a function of many variables, and this function determines the limit and degree of rheology effect on the results of calculations. For the first time this pattern takes into account the heterogeneity of the rheological characteristics of rocks and the specifics of the computational domain geometry. The use of the obtained patterns makes it possible to simplify significantly the process of modelling in time and space of the geomechanical systems, which have a complex structure describing the interaction of several objects.
\end{abstract}

\section{Introduction}

The decline in global underground coal production is primarily due to the low level of energy efficiency. In the absence of global industrial economy growth and an increase in the availability of other energy resources, underground coal mining becomes uncompetitive. Low indicators of self-recoupment and the high risks associated with ensuring the stable coal enterprises operation do not permit a policy of innovative development, which can provide a significant increase in energy efficiency $[1,2]$.

In such conditions, the most effective method for maintaining a sufficient level of coal mining is possible only due to the local optimizations of the production process with the design and service of coal mining enterprises. Most technological chains of the mine depend to a greater or lesser extent on the state of the rock massif within which the preparatory and stope face mine workings are operated $[3,4,6,11]$.

\footnotetext{
*Corresponding author: vvlapko@gmail.com
} 
At the moment there are various methods for determining the state of the rock massif geomechanical systems and mine workings based on the full-scale, laboratory and computational experiments $[8,10]$. But any method has its own accuracy limit of the obtained results and, thus, it has different levels of complexity in carrying out the experiments themselves. The recommendations received as a result of the conducted studies should ensure the effective operation of the mine. Therewith, the costs of the experiments themselves should be minimal.

The simplest way to reduce the cost for behaviour prediction of a structurally complex system with a wide range of possible description parameters is the segmentation of the description system of the studied objects in order to simplify the procedure for obtaining the results $[5,9]$. Such an approach allows using different description types of physical object or process within the single methodology framework, depending on the requirements made during the research.

The investigation of the stress-strain state of breakage face geomechanical system, as a rule, is held in order to obtain technical solutions to minimize the deformations on the contours of the preparatory and stope face mine workings. It is a general practice here to perform estimative calculations by the simplified schemes. As a result, the adopted technological solutions are either excessive in their characteristics, or do not provide sufficient operational characteristics [7]. It turns out that the total costs for ensuring production simply do not justify engineering work.

The main objects, the parameters of which have a wide range of possible values, are the rock layers. These parameters are controlling ones for the determination of acceptable technological solutions on operation with a specific geomechanical system. Thus, the complexity of the simulation model and its adequacy is directly dependent on the method of the laminal rock massif description.

Over time, the growth in the mine working contour deformation causes a change in the mechanical characteristics of the limited volumes of rocks. This induces the formation of main cracks, which, in combination with water-cut, fracturing and tectonic stresses, modifies the initial conditions of mine working operation. Therefore, when carrying out a computational experiment, one must resort to the use of various methods for these characteristics description. An account in the rheology calculations allows to make a comprehensive description of the influence of such physical features of rocks on the deformation of the mine working contour. However, a rapid increase in computational costs during the computational experiments requires the determination of the effective range in the application of this modelling type.

\section{Specifics of simulation models formation and stages of computational experiment realization}

In this study, a computational experiment was based on the finite element method implemented in the ANSYS software package.

In general view, the geomechanical model of the breakage face has been implemented as a stack of parallelepipeds with different heights, but with the same width and length of the base. Each parallelepiped is used to describe the geometry of a certain rock layer. In this model of the rock massif, three cuttings are made, which represent the preparatory and stope face mine workings, and the space that arises after the collapse of the breakage face immediate roof. The geometric model of the computational domain was fixed along the lateral borders of the coal seam from the side of virgin rock massif. The loadings were applied on the upper and lower borders of the compound parallelepiped.

The impact on the rock massif of the preparatory mine working support and extracting equipment working bodies was modelled as a combination of forces. The mechanized 
support was modelled by a set of parallelepipeds in accordance with the real objects dimensions. When performing the calculations, a bench was modelled with a depth of $0.3 \mathrm{~m}$ on the breakage face plane. This bench indicates the location of the working body of the plough or cutter-loader.

When performing calculations, the parameters of five materials were used: steel, coal, argillite, siltstone and sandstone. These parameters were selected as averaged characteristics of rocks with different strength properties. The curves of rheological increments were constructed for each material (Fig. 1).

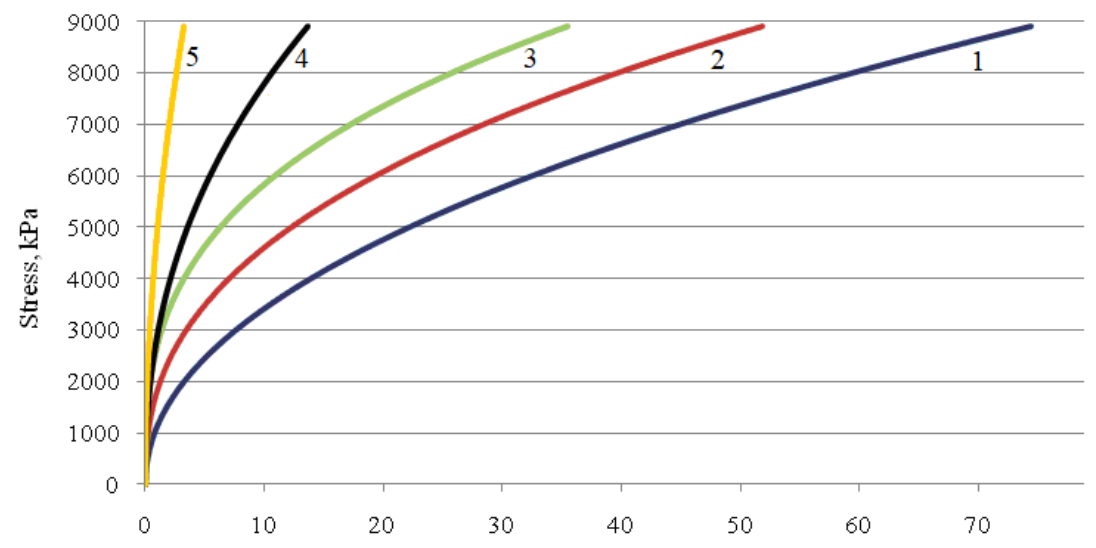

Fig. 1. Curves of rheological deformations increment for various materials of the simulation model: 1 - steel; 2 - coal; 3 - argillite; 4 - siltstone; 5 - sandstone.

These curves for coal and rocks were obtained in two stages. At the first stage, laboratory tests were carried out to determine the deformation characteristics of the test samples of materials. Conducting a study on the rheology effect on the stress-strain state of the breakage face geomechanical system suggests the calculation of deformations on a time interval of only 80 hours. Therefore, the tests of coal and rock samples were carried out in real time, and this greatly simplified the procedure of the experiment.

At the second stage, the laboratory test results were subjected to statistical processing to identify the averaged parameters of the deformation characteristics change of the selected materials. The obtained curves of total deformation had to be decomposed on the basis of the superposition principle into the elastic-plastic deformation curve and the curve of deformation under the rheology effect. The last one has already been used in the basic calculations of a computational experiment.

During the computational experiment, all calculations were carried out in an elasticplastic setting without or with regard to rheology. The materials of rock layers' models, the velocity of the combined machine, which moved along the breakage face plane and the velocity of the breakage face displacement deep into the virgin rock massif varied in the calculations.

In total, there were performed 43 basic calculations out of 81 planned ones. The change in the number of calculations is due to the peculiarities of the obtained results. So, for example, from the analysis of the curves of already performed calculations it became obvious that changes in the rock layers' parameters, which were modelled second from above and from below, do not effect on the contour deformations of the studied mine workings. Thus, the calculations were cancelled, the performance of which could not give serious SSS deviations of the geomechanical system. 


\section{Analysis of the obtained data and formation of the identified patterns description}

The basis for further research was to analyse the stresses intensity and linear deformations pattern in the area of junction of the preparatory and stope face mine workings. For most of calculation results by finite element method, the main differences were concentrated in the computational domain restricted to $30 \mathrm{~m}$ along the breakage face, $60 \mathrm{~m}$ along the axis of the preparatory mine working and $15 \mathrm{~m}$ in height. The centre of this area was $2 \mathrm{~m}$ behind the mechanized support model in the mined-out space, $6 \mathrm{~m}$ above the coal seam and $11 \mathrm{~m}$ from the longitudinal axis of the preparatory mine working from the side of the breakage face.

As the standard, four calculations were made in an elastic-plastic setting with different combinations of mechanical characteristics of the rock layers and with two different velocities of the breakage face plane displacements. An illustration of the results of one of the calculations is given for two cross-sections of three dimensional curves of stresses intensity (Fig. 2).

$a$

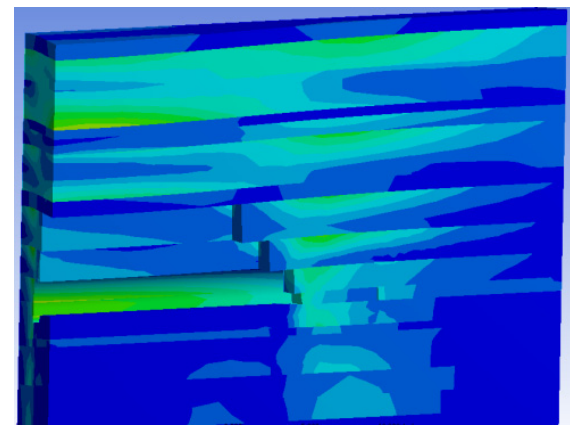

$b$

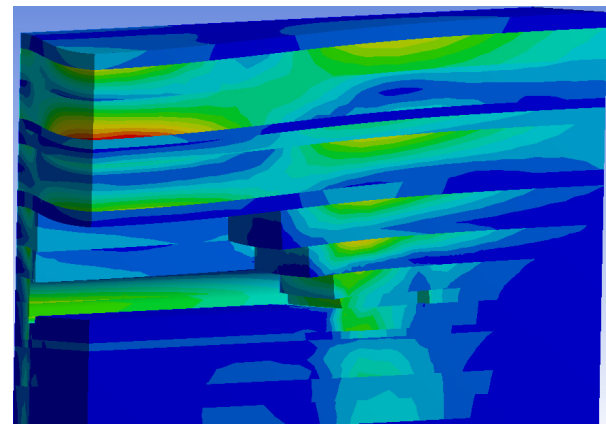

Fig. 2. Stresses intensity distribution in vertical planes directed along the axis of preparatory mine working and located in relation to it depthward into the stope face mine working at a distance of $\mathrm{a}-8 \mathrm{~m}$ and $\mathrm{b}-16 \mathrm{~m}$.

The analysis of the presented figures shows that with this variant of calculation, above and below the mechanized support, a zone of increased rock pressure is formed, the value of which increases toward the breakage face. In this case, the velocity of displacement of the breakage face plane increases the geometric size of this zone, but does not change the maximum values of stresses intensity.

The stresses pattern presented in Fig. 2a shows that in the rock massif area, which is directly adjacent to both sides of the preparatory mine working, the stresses concentration has one order. For all variants of calculations this pattern does not change. It turns out that, for both soft and robust rocks, without taking rheology into account, the contour deformation forecast of the preparatory mine working has one kind and a relatively narrow range of absolute values variation. For the considered variants of calculations, with a variation of the deformation characteristics within $82 \%$, the absolute deformations varied within $23 \%$.

The stresses intensity distribution in Fig. $2 b$ corresponds to the cross-section through which the bench has already passed, which models the contact surface with the working body of the cutter-loader. When comparing the stresses distribution in the virgin massif near the breakage face plane in Fig. 3a, b, we get a stresses drop in the bottom, an increase of the stresses intensity in the rock massif in front and above the breakage face plane. This 
peculiarity is manifested most intensively with a combination of high velocity of the cutterloader moving and the occurrence of soft rocks in the roof and bottom of the stope face mine working.

Three specific cross-sections of stresses intensity curves in Fig. 3 show the SSS main features of the breakage face geomechanical system in calculations performed with account of the rheology curves presented in Fig. 1.

$a$

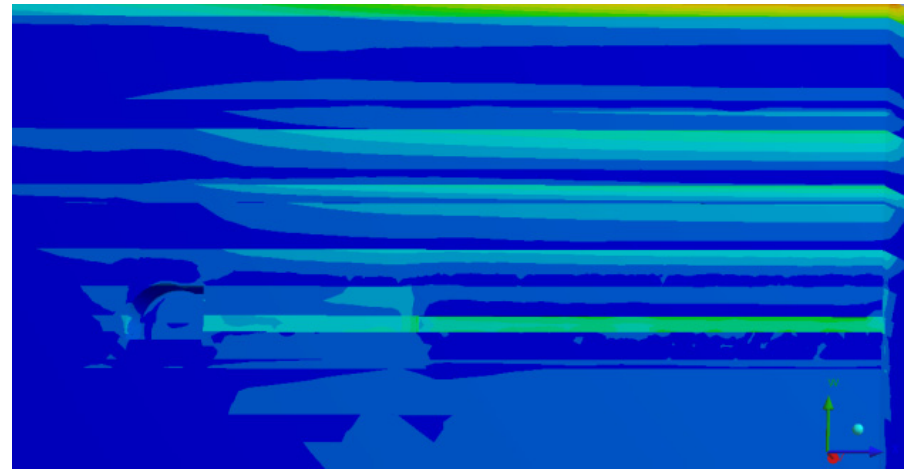

$b$

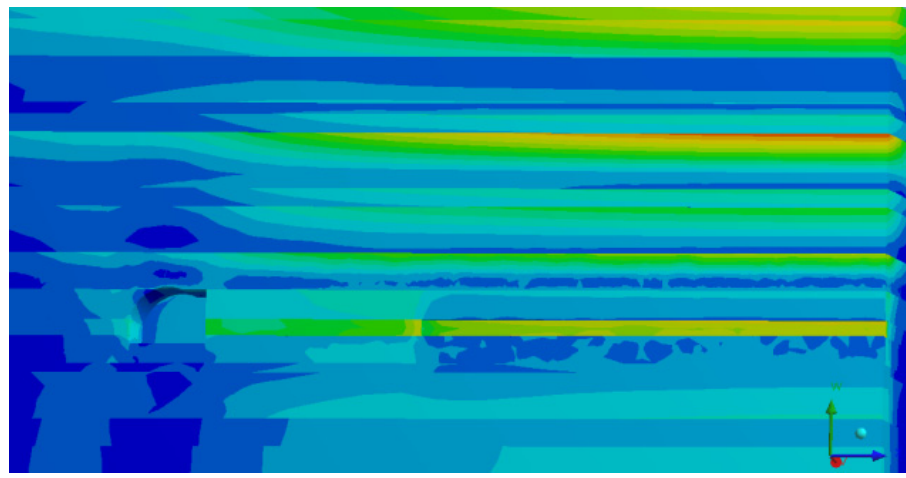

$c$

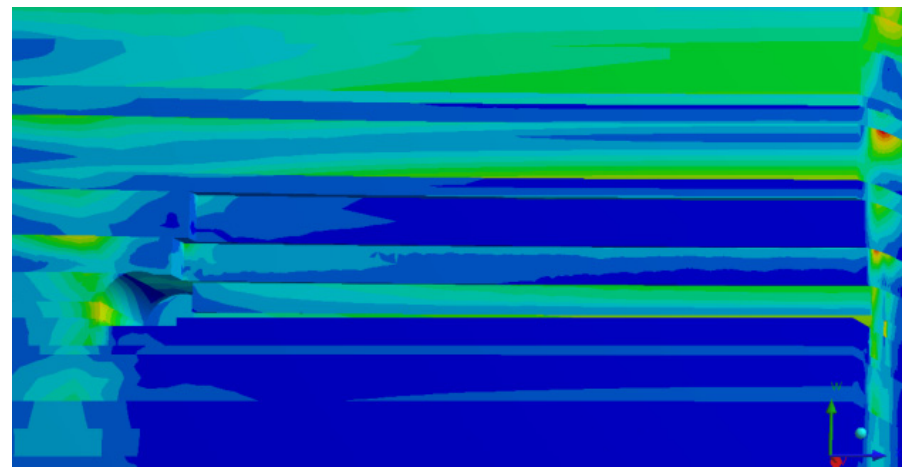

Fig. 3. The stresses intensity field in a geomechanical system along the cross-section: a - at a distance of $5 \mathrm{~m}$ in front of the breakage face plane; $\mathrm{b}$ - along the breakage face plane; $\mathrm{c}-$ at $60 \mathrm{~m}$ behind the breakage face. 
When considering the cross-section located in the virgin rock massif in front of the breakage face plane (Fig. 3a), there are two main peculiarities of the obtained results. The first peculiarity - soft rocks are stressed by $1.5-2$ times less than rocks with higher strength characteristics. The second peculiarity - with the approach of the bench formed by the working body of the cutter-loader, a 'wave' of stresses intensity is formed in the coal seam and the adjacent rock layers to the longitudinal axis of the extraction working for a distance of three widths (about $15 \mathrm{~m}$ ). This effect is more manifested when the mechanical characteristics of the rock layers are alternated. The absolute values of the mechanical parameters of rock layers have only quantitative effect on the stresses intensity change.

In the cross-section, which coincides with the breakage face plane (Fig. 3b), the stresses intensity in the coal seam has the maximum values. The deformation of the preparatory working contour is still insignificant and does not exceed $0.3 \mathrm{~m}$. In general, the evolution of the stresses pattern is observed, at which the rheological deformations growth is not yet observed. The drop in stresses in the rock layers adjacent to the coal seam indicates the beginning of formation of the unloading centres and the changeover of the simulation model part to the limiting state.

When analysing the velocity effect of the breakage face plane displacement and the bench formed by the working body of the cutter-loader we obtain the following: the first is that a high velocity of the bench displacement leads to significant (up to $37 \%$ ) differences in stresses intensity in one rock layer; the second - an increase in the velocity of the breakage face plane displacement determines the velocity of total deformations of the mine working contour, but in a certain range and in case of a large spread in the values of the rock layers strength parameters; third - the considered combination of displacements works on the principle of mutual effect, that is, there is a stress intensity minimization in the coal seam and rock layers at certain velocity pairs.

Fig. 3c shows a development of rheological deformations in the rock massif in combination with the stresses intensity decrease after the collapse of the breakage face immediate roof. A zone of increased stresses is formed in the left side of the preparatory mine working. This peculiarity is manifested for all calculations using the rheology curves. Thus, when considering the rheology, the preparatory working support is under effect of a second wave of increased rock pressure over time after removing the breakage face plane from it. The stresses values in the zone of increased rock pressure depend on the combination of mechanical parameters of rock layers. The distance from the breakage face plane, on which this zone occurs, is directly proportional to the velocity of the breakage face displacement.

For the calculation, the results of which are presented above (Fig. 3), we analyse the deformation change in one of the selected points. In Fig. 4 the curves are shown of the displacement of the point located in the zenith of the preparatory mine working arch. At the initial time, this point was located in the plane of the breakage face. In the considered variant, the average velocity of the breakage face plane displacement was $1 \mathrm{~m} / \mathrm{hr}$.

The considered curves of total deformations in the point of the simulation model take into account the rheology of rocks and, at the same time, have one peculiarity, which is manifested only at the elastic-plastic calculation. If the deformation values along the axis of the preparatory mine working do not exceed $0.05 \mathrm{~m}$ and remain in the elastic zone, then the increase in vertical deformation is by an order greater and the pattern of its changes has an inflection point.

Such a peculiarity is observed not for all variants of computational experiment calculation. In addition, in one calculation for certain rock layers, two variants for changing vertical deformations can be realized. In the first variant, the deformation change corresponds to the characteristic presented in Fig. 4. In the second, the change in deformation occurs according to a power-law pattern without any peculiarities of the curve $[12,13]$. 


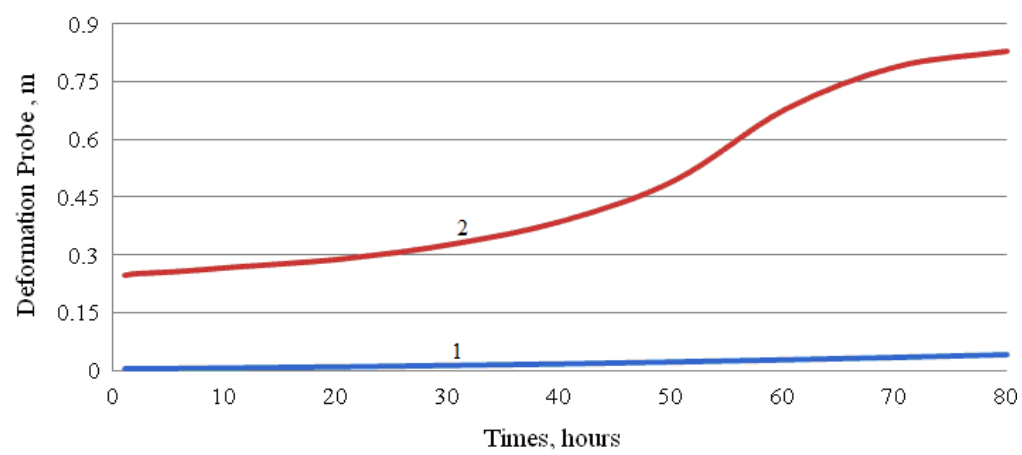

Fig. 4. Linear deformations in the selected point: 1 - directed along the axis of the preparatory mine working; 2 - vertical.

Thus, based on the data obtained by the deformations analysis in the calculation points of the model, an expression was obtained that describes the condition of necessity to take into account rheology depending on the number of layers and their deformation characteristics:

$$
\frac{N \cdot \Delta \varepsilon_{\max }}{11.41\left|\Delta \bar{\varepsilon}_{i}-\Delta \bar{\varepsilon}_{j}\right|}<1,
$$

where $N$ is the number of modelled layers; $\Delta \varepsilon_{\max }$ is the maximum increment of deformations in the computational domain; $\Delta \bar{\varepsilon}_{i}$ and $\Delta \bar{\varepsilon}_{j}$ are incremental matrices according to the layers of the model, where $i=\overline{1, N-1}$ and $j=\overline{2, N}$.

Using only the expression (1) to determine the range of calculations with account of rock layers' rheology does not allow to consider the dynamics of changes in time of the geometric characteristics of the simulation model. To account for these peculiarities of the computational experiment, it was decided to analyse the change in potential energy of deformation in the studied computational domain (Fig. 5).

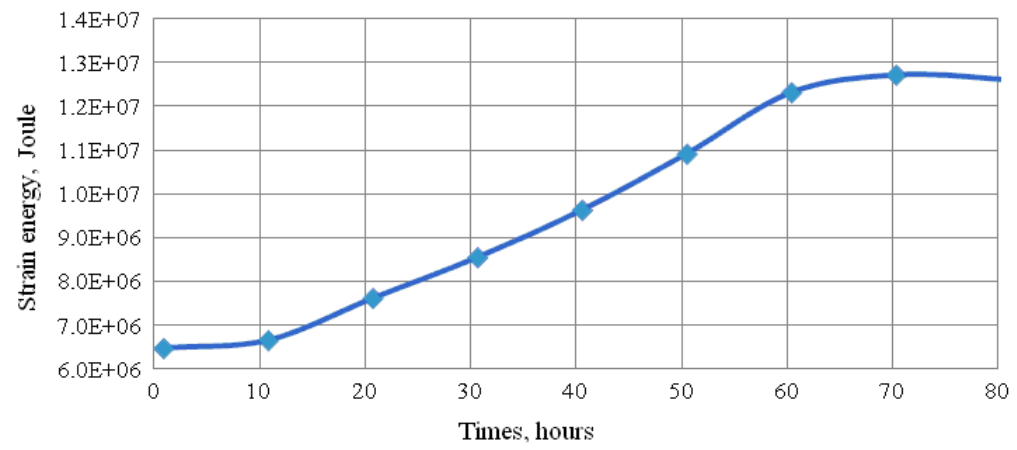

Fig. 5. The change of the deformation energy in calculations performed with account of rheology.

The curve of deformation energy change presented in Fig. 5, shows that taking into account rheology leads to energy dissipation when reaching a certain level of SSS calculated values in the chosen volume of the model. The analysis of similar curves for various combinations of the displacement velocities of the rock bench and the breakage face plane has shown that there is a limit beyond which the effect of rheology of rocks on 
the calculation result becomes minimal.

We obtained an expression relating the velocities of the rock bench and breakage face plane with deformations occurring in selected points of simulation model:

$$
\frac{V_{x}}{14.8 V_{z}} \cdot \frac{P_{t} \cdot \bar{\varepsilon}_{n \cdot t}}{\bar{U}_{n \cdot t}}>5.12,
$$

where $n$ is the number of a rock layer; $V_{x}$ and $V_{z}$ are velocities of displacement of the bench in the coal seam and the breakage face plane, respectively; $P_{t}$ is the external pressure on the system at the selected time; $\bar{\varepsilon}_{n \cdot t}$ and $\bar{U}_{n \cdot t}$ are matrices of deformations and deformation energy, describing the state of the computational domain in the rock layer $n$ and at the time of calculation $t$.

The systems of equations described in expressions (1) and (2) together determine the range of parameters of the simulation model of the breakage face geomechanical system, in which it is necessary to use the rheological characteristics of rocks in a computational experiment.

\section{Conclusions}

The obtained results of the computational experiment have shown that the effect of the rheology of rocks on the distribution of stresses and deformations in the breakage face geomechanical system has a different value and depends on two main factors - the structure of the rock massif and the velocity of the breakage face plane displacement.

There are upper and lower limits of the geometric and physical complexity of the simulation model description for which rheology accounting does not affect the quality of modelling or the conditions for a computational experiment performance. This makes it possible to neglect rheology indices even in calculations of structurally complex geomechanical systems.

The change in the stress-strain state of the breakage face geomechanical system under the effect of rheology of rocks increases in direct proportion with the increase in the number of rock layers which have different rheological parameters. It has a power-law dependence on the increase in the difference in time of deformations for different rock layers, and depends on the ratio of the averaged velocity of the breakage face plane displacement and the velocity of displacement of the rocks bench formed by the working body of the cutter-loader.

Thus, the results of these studies make it possible to provide acceptable indicators of the computational experiment quality in determining the optimal technological parameters of the geomechanical system in a context of minimizing the costs for calculations and subsequent mine workings operation. The resulting economic effect compared to traditional approaches can be up to $12 \%$.

The authors express their gratitude to the staff of Department of Underground Mining of the National Mining University for the multifaceted assistance.

\section{References}

1. Barabash, M.V., \& Cherednichenko, Yu.Ya. (2015). Transformation SHC "Pavlogradvugillia" in the world class coal-mining company - PJSC "DTEK Pavlogradvugillia". Mining of Mineral Deposits, 9(1), 15-23. https://doi.org/10.15407/mining09.01.015 
2. Fomychov, V. (2016). Efficiency of energy resource production while optimizing parameters of socio-economic balance. Mining of Mineral Deposits, 10(1), 89-95. https://doi.org/10.15407/mining10.01.089

3. Bondarenko, V., Kovalevs'ka, I., \& Fomychov, V. (2012). Features of carrying out experiment using finite-element method at multivariate calculation of mine massif - combined support system. Geomechanical Processes During Underground Mining, 7-13. https://doi.org/10.1201/b13157-3

4. Fomychov,V. (2012). Premise development of clearing models of the frame anchor support at nonlinear characteristics physics mediums behavior. Naukovyi Visnyk Natsionalnoho Hirnychoho Universytetu, (3): 45-52.

5. Fomychov, V., \& Sotskov, V. (2018). Determination of parameters of non-uniform fractured rock massif in computing experiment. Journal of Geology, Geography and Geoecology, 26(1), 26-32. https://doi.org/10.15421/111803

6. Małkowski, P., Niedbalski, Z., \& Majcherczyk, T. (2016). Roadway design efficiency indices for hard coal mines. Acta Geodynamicaet Geomaterialia, 13(2): 201-211.

7. Meng, L., Nan, Z., Jixiong, Z., \& Zhicheng, L. (2017). Numerical Modelling of Mechanical Behavior of Coal Mining Hard Roofs in Different Backfill Ratios: A Case Study. Energies, 10(7), 1-18. https://doi.org/10.3390/en10071005

8. Fomichov, V., Sotskov, V., \& Malykhin, A. (2014). Determination and analysis of the acceptable benchmark changes of the stress strain state of frame and bolt fastening elements of dismantling drift when approaching a working face. Naukovyi Visnyk Natsionalnoho Hirnychoho Universytetu, (1), 22-26.

9. Kovalevska, I., Illiashov, M., Fomychov, V., \& Chervatuk, V. (2012). The Formation of the Finite-Element Model of the System "Undermined Massif - Support of Stope". Geomechanical Processes During Underground Mining: School Underground Mining 2012, 73-79. https://doi.org/10.1201/b13157-13

10. Rosenblatt, J., \& Bell, S. (1999). Mathematical Analysis for Modeling. Boca Raton.

11. Sotskov, V.O., Demchenko, Yu. I., Salli, S.V., \& Dereviahina, N.I. (2017). Optimization of parameters of overwoked mining gallery support while carrying out long-wall face workings. Naukovyi Visnyk Natsionalnoho Hirnychoho Universytetu, (6), 34-40.

12. Zienkiewicz, O., Taylor, R. (2000). The Finite Element Method. Vol. 1: The Basic. Oxford: Butterworth-Heinemann.

13. Fomichov, V., Sotskov, V., Pochepov, V., \& Mamaikin, O. (2018). Formation of a calculation model determining optimal rate of stoping face movement with a large deformation of a rock massif. ARPN Journal of Engineering and Applied Sciences, 13(7), 2381-2389. 